\title{
Users' Preference Prediction of Real Estates Featuring Floor Plan Analysis using FloorNet
}

\author{
Naoki Kato \\ The University of Tokyo \\ Bunkyo Ku, Tokyo, Japan \\ kato@hal.t.u-tokyo.ac.jp \\ Kiyoharu Aizawa \\ The University of Tokyo \\ Bunkyo Ku, Tokyo, Japan \\ aizawa@hal.t.u-tokyo.ac.jp
}

\author{
Toshihiko Yamasaki \\ The University of Tokyo \\ Bunkyo Ku, Tokyo, Japan \\ yamasaki@hal.t.u-tokyo.ac.jp \\ Takemi Ohama \\ Ietty Co., Ltd. \\ Shibuya Ku, Tokyo, Japan
}

\begin{abstract}
In recent years, with the progress of e-commerce, recommendation for not only mass-produced daily items, such as books, but also special items that are not mass-produced has become an important task. In this study, we present an algorithm for real estate recommendation. There are no identical properties in the world, properties already occupied by someone else cannot be recommended, and users rent or buy properties only a few times in their lives. Therefore, automatic property recommendation is one of the most difficult tasks. In this study, we predict users' preference for properties, which is the first step of property recommendation, by combining content-based filtering and multilayer perceptron (MLP). In the MLP, we used not only attribute data of users and properties but also the deep features extracted from floor plan images of properties. As a result, we succeeded in predicting users' preference with an accuracy of $60.7 \%$.
\end{abstract}

\section{CCS CONCEPTS}

- Information systems $\rightarrow$ Recommender systems; • Computing methodologies $\rightarrow$ Neural networks;

\section{KEYWORDS}

floor plan, machine learning, prediction, preference, real estate, recommendation

ACM Reference Format:

Naoki Kato, Toshihiko Yamasaki, Kiyoharu Aizawa, and Takemi Ohama. 2018. Users' Preference Prediction of Real Estates Featuring Floor Plan Analysis using FloorNet. In Multimedia for RETech'18: Workshop on Multimedia for Real Estate Tech, June 11, 2018, Yokohama, Japan. ACM, New York, NY, USA, 5 pages. https://doi.org/10.1145/3210499.3210525

\section{INTRODUCTION}

In recent years, with the expansion of online services, e-commerce users have increased. Recommendation of goods is often exercised

Permission to make digital or hard copies of part or all of this work for personal or classroom use is granted without fee provided that copies are not made or distributed for profit or commercial advantage and that copies bear this notice and the full citation on the first page. Copyrights for third-party components of this work must be honored. For all other uses, contact the owner/author(s).

Multimedia for RETech'18, June 11, 2018, Yokohama, Japan

(c) 2018 Copyright held by the owner/author(s).

ACM ISBN 978-1-4503-5797-5/18/06.

https://doi.org/10.1145/3210499.3210525 on e-commerce websites, and its accuracy has improved owing to the recent increase in data. There are two major types of recommender systems: content-based filtering and collaborative filtering proposed by Goldberg et al. [3]. However, these recommender systems can be used only when items are mass-produced. When almost every item of a category is unique, such as in real estate properties, it is difficult to recommend the item automatically.

Under such circumstances, a real estate technology called Real Estate Tech (RETech) began to grow rapidly, and the Ministry of Land, Infrastructure, Transport and Tourism (MLIT) ${ }^{1}$ in Japan conducted social experiments to deregulate activities on the Internet in the real estate industry ${ }^{2}$. Therefore, property recommendation on websites has become an important task.

This study aims at implementing a recommender system for special data that the general recommender systems described above cannot be used for, i.e., real estate property. We predict users' preference for properties, which is the first step of property recommendation, using the dataset that includes users' evaluations for properties possessed by $\mathrm{Ietty}^{3}$, which is a rental company of real estate properties. Generally, users search for desired properties on property search websites and contact the real estate companies possessing the properties. However, Ietty recommends properties to the users on its website, and the users evaluate the properties online, after which they preview the properties or lease them. Thus, if an effective method for prediction of users' preference for properties using their evaluation data is available, property recommendation can be performed more easily.

Accordingly, we proposed a prediction system combining contentbased filtering and multilayer perceptron (MLP) to predict users' preference for properties. Moreover, we used deep features of floor plans as input of the MLP to improve accuracy. Consequently, we succeeded in predicting users' preference for properties with an accuracy of $60.7 \%$.

The remainder of this study is organized as follows. In section 2 , we discuss the related works about recommendation systems and analysis of floor plan images. In section 3, we describe our proposed method in detail. In section 4 , we show metrics of the main experiments and the results. In section 5, we conclude this study.

\footnotetext{
$\overline{{ }^{1} \text { http://www.mlit.go.jp/en/index.html }}$

${ }^{2}$ http://www.mlit.go.jp/totikensangyo/const/sosei_const_tk3_000092.html

${ }^{3}$ https://ietty.me/
} 


\section{RELATED WORKS}

\subsection{Recommendation Systems}

Collaborative filtering is classified into two types: memory-based collaborative filtering proposed by Goldberg et al. [3] and modelbased collaborative filtering proposed by Breeze et al. [1]. In memorybased collaborative filtering, the users' purchase/evaluation data of items are stored in a memory, and collaborative filtering is performed using these stored data every time recommendation is required. However, model-based collaborative filtering first develops a model that predicts items with high probability of being purchased/evaluated by the users, and collaborative filtering is performed using this model when recommendation is required. In addition, Pennock [13] presented hybrid collaborative filtering by combining these two methods, and Xue [18] improved the performance of hybrid collaborative filtering by data interpolation using clustering.

In the Netflix Prize competition ${ }^{4}$, Simon [2] greatly improved the performance of recommender systems by using the matrix factorization algorithm. The matrix factorization algorithm finds vectors of both user factors $p_{u}$ and item factors $q_{i}$ satisfying (1). $\kappa$ is a set of $(u, i)$ (a pair made up of user $u$ and item $i$ ), whose evaluation value $r_{u i}$ exists in the train set, and $r_{u i}=q_{i}^{T} p_{u}$ is the predicted evaluation value of item $i$ for user $u$ in the test set.

$$
\min _{q, p} \sum_{(u, i) \in \kappa}\left(r_{u i}-q_{i}^{T} p_{u}\right)^{2}+\lambda\left(\left\|q_{i}\right\|^{2}+\left\|p_{u}\right\|^{2}\right)
$$

Simon [2] solved this optimization problem using stochastic gradient descent (SGD) optimization by iterating (2)-(4).

$$
\begin{aligned}
e_{u i} & =r_{u i}-q_{i}^{T} p_{u} \\
q_{i} & \leftarrow q_{i}+\gamma \cdot\left(e_{u i} \cdot p_{u}-\lambda \cdot q_{i}\right) \\
p_{u} & \leftarrow p_{u}+\gamma \cdot\left(e_{u i} \cdot q_{i}-\lambda \cdot p_{u}\right)
\end{aligned}
$$

Koren et al. [8], who won the Netflix Prize, introduced both SGD and alternating least squares (ALS) optimizations, which alternately optimized $q_{i}$ and $p_{u}$, and they highlighted that matrix factorization overcame the curse of dimensionality caused by high-dimensional data and achieved highly accurate recommendations even for highdimensional data.

Hidasi et al. [6] proposed a recommender system using users' data of all their past clicks on an e-commerce website with the gated recurrent unit (GRU) model, which is a type of modern recursive neural network (RNN). However, all these techniques described above did not work well for highly sparse data. Further, for the existing automatic recommender systems for real estate properties, such as Ietty, the system applies a rule-based algorithm that realizes users' desired conditions.

\subsection{Floor Plan Image Analysis}

The studies on floor plans of properties before the development of deep learning were based on analyzing floor plans graphically. Hanazato et al. [4] analyzed floor plans using adjacency graphs with rooms, corridors, etc., as labeled nodes and four sampled datasets that divided the floor plans according to their square measures. They examined patterns and their numbers of the adjacency graphs,

\footnotetext{
${ }^{4}$ https://www.netflixprize.com/
}

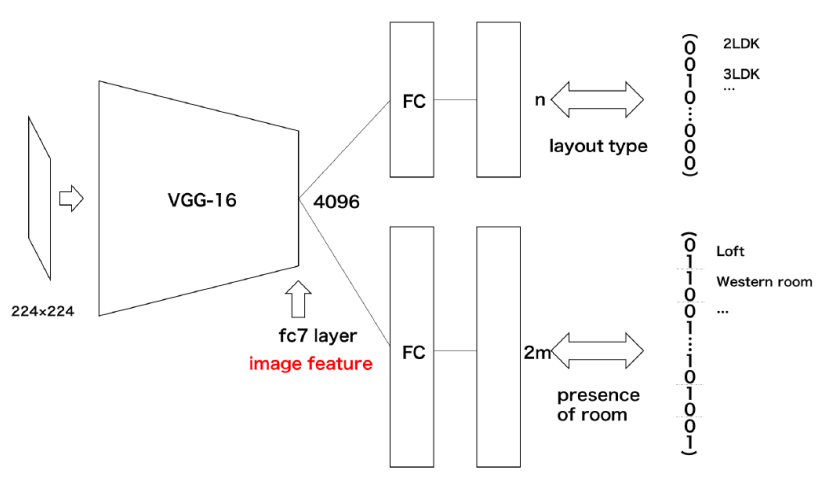

Figure 1: Takada's network architecture [16]

and classified them into six types according to the distances from each node to other nodes of the adjacency graphs. In addition, Takizawa et al. [17] analyzed the rent of properties using adjacency graphs with rooms, corridors, etc., as labeled nodes and doors, glasses, etc., as labeled edges, and floor plans of 3LDK, 3K, or 3DK apartment in Kyoto, Japan. They extracted subgraphs from the adjacency graphs and effectively estimated the rent from the presence/absence of common subgraphs. However, the cost of creating adjacency graphs of floor plans is very high.

Ohara et al. [12] developed a property search system that reflected users' preference using common subgraphs of floor plans. Takada et al. [16] used the dataset created by Ohara et al. [12] to estimate graphs of floor plans and retrieved similar floor plans with a query floor plan. They fine-tuned a model from the ImageNet [9] pre-trained model by multi-task learning (layout type such as one bedroom + one bathroom and presence of the room like loft, western room, etc.) using the network shown in Fig. 1, and solved the retrieval task using deep features extracted from the floor plan images by the model. Hereafter, we call Takada's model as FloorNet.

\section{PROPOSED METHOD}

\subsection{Dataset}

Ietty, a rental company of real estate properties, has attribute data of real estate properties and users, and recommends several properties to the users by the rule-based algorithm on its website. Further, the users can evaluate each recommended property, selecting "want to see the property," "register as favorite," or "no interest." In this study, we obtained the evaluation data for a period between 2016-2017 which contains 220,094 cases with the floor plan images, and divided them into train set, validation set, and test set at a ratio of 3: 1: 1 . Thus, the dataset includes attribute data of 19,538 users, and attribute data and floor plan images of 131,947 properties.

\subsection{Overview of Proposed Method}

We define "want to see the property" and "register as favorite" as positive evaluation and "no interest" as negative evaluation of properties by the users, designate these as users' preference for properties, and predict it using methods available for sparse data. 
In this study, we predict preference by content-based filtering using similarities of both users and items, and MLP that adds the deep features extracted from floor plan images using FloorNet into the input. Because these methods are available without past evaluation data by the users to be recommended items, they are robust to sparse data. We also propose a system called hybrid filtering that combines these two methods and predict users' preference by it.

Although the layout type of floor plans, such as one bedroom + one bathroom is included in the attribute data of properties, it is expected that the accuracy of preference prediction is improved by considering the floor plans. For example, in the same layout type, some users desire properties that prevents a direct connection between an entrance and a child's room so that young children do not come out outside without their parents' permission, while other users desire convenient properties that allows walking out into the corridor from each room. Therefore, we propose adding deep features of floor plans into the input of MLP to consider such floor plans. The details of each method are described in the following sections.

\subsection{Content-based Filtering}

We predict preference by content-based filtering using similarity of attributes for both users and properties. We define $u$ and $i$ as the attribute data of a user and a property, respectively, and let the positive evaluation value be $w$ (the ratio between the number of the negative evaluations and the number of the positive evaluations in the train set) and the negative evaluation value be -1 . Using a pair $(u, i)$ in the train set whose evaluation value $r_{u i}$ is known and a pair $\left(u_{\text {test }}, i_{\text {test }}\right)$ in the test set, we calculate the cosine similarity $\cos \left(u_{\text {test }}, u\right)$ for each $u$, and let $U_{C B F}$ be the set of top $k_{u} \%$ of all $u$ by similarity. Similarly, we calculate the cosine similarity $\cos \left(i_{\text {test }}, i\right)$ for each $i$, and let $I_{C B F}$ be the set of top $k_{i} \%$ of all $i$ by similarity. Further, we calculate the predicted evaluation value $v_{C B F}$ for the pair $\left(u_{\text {test }}, i_{\text {test }}\right)$ in the test set as (5).

$$
v_{C B F}=\sum_{u \in U_{C B F}} \sum_{i \in I_{C B F}} \cos \left(u_{\text {test }}, u\right) \cdot \cos \left(i_{\text {test }}, i\right) \cdot r_{u i}
$$

We predict the preference as positive when the predicted evaluation value $v_{C B F}$ is larger than zero and predict it as negative otherwise.

\subsection{MLP}

We predict preference using the MLP, whose network architecture is shown in Fig. 2. We use the attribute data of users and properties, and the deep features extracted from image data of properties as input of the MLP. Moreover, we improve generalization by using dropout [15] and batch normalization [7]. At the time of training, we use the train set and the validation set, and at the time of testing, we exclude the dropout and use the test set. In the final fully connected (FC) layer, a two-dimensional vector $\left(x_{n}, x_{p}\right)$ corresponding to negative and positive evaluation is obtained as the output, and we predict the preference as positive when the predicted evaluation value $v_{M L P}=x_{p}-x_{n}$ is larger than zero, and predict it as negative otherwise.

To extract deep features of floor plan images, we use a model fine-tuned by the improved method of Takada et al. [16], FloorNet. In detail, we use ResNet50 [5] instead of VGG-16 [14] and use the

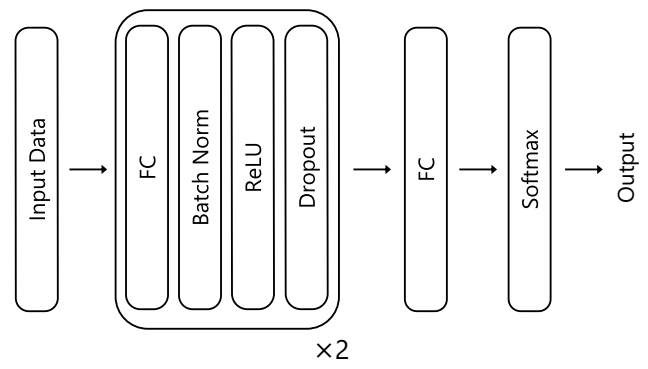

Figure 2: Network architecture of the MLP

floor plan images randomly rotated as input for data augmentation. The dataset we use for fine-tuning is the same as that used by Takada et al. [16], which was created by Ohara et al. [12] and contains floor plan images from two sources: SUUMO 5 and HOME'S dataset ${ }^{6}$. We use the fine-tuned model as an extractor and obtain the feature vectors of 2,048 dimensions of the pool5 layer as deep features of floor plans.

\subsection{Hybrid Filtering}

We predict preference by weighted sum of predicted evaluation value $v_{C B F}$ in 3.3 and $v_{M L P}$ in 3.4. An overview of the architecture is shown in Fig. 3. We use the validation set instead of the test set in 3.3 and 3.4, then calculate standard deviations $\sigma_{C B F}$ and $\sigma_{M L P}$ of predicted evaluation values for each method, and designate reciprocal numbers of them as $w_{C B F}$ and $w_{M L P}$, which are weights to be added to each predicted evaluation value for the test set as (6).

$$
v_{H F}=w_{C B F} \cdot v_{C B F}+w_{M L P} \cdot v_{M L P}=\frac{v_{C B F}}{\sigma_{C B F}}+\frac{v_{M L P}}{\sigma_{M L P}}
$$

By performing such scaling, we obtain the predicted evaluation value $v_{H F}$, which emphasizes the two methods to the same extent, and like other methods, we predict preference as positive when the predicted evaluation value $v_{H F}$ is larger than zero, and predict it as negative otherwise.

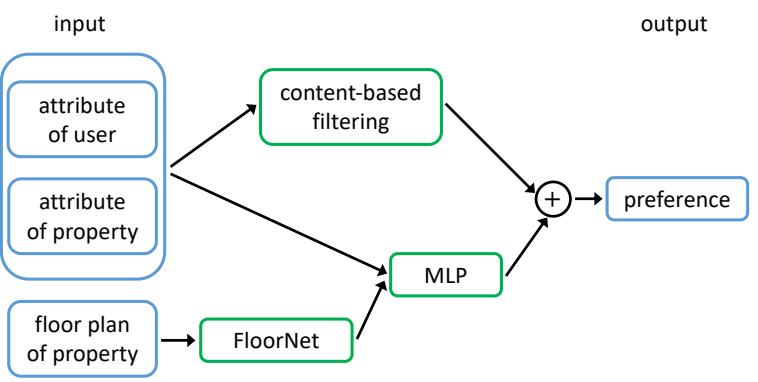

Figure 3: Architecture of the hybrid filtering

\footnotetext{
${ }^{5}$ http://suumo.jp/

${ }^{6}$ http://www.nii.ac.jp/dsc/idr/next/homes.html
} 


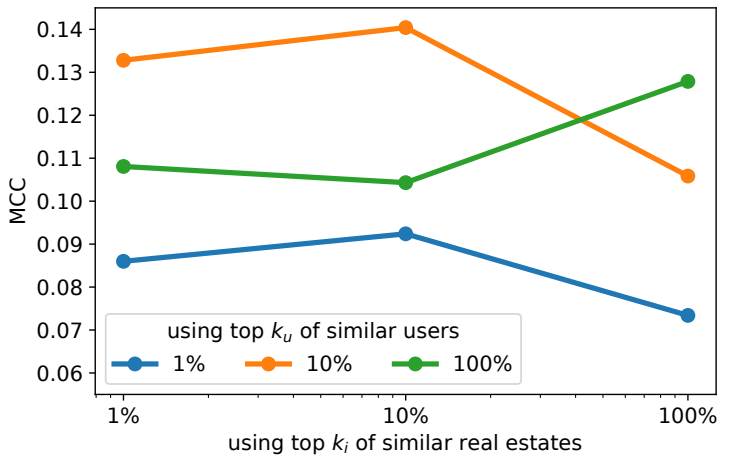

Figure 4: Prediction results of users' preference for properties by the contents-based filtering

\section{EXPERIMENTS}

\subsection{Metric}

There are 76,871 positive data (35\%) and 223,223 negative data $(65 \%)$ in the total 220,094 evaluation data. There is a bias in the dataset; thus, we used the Matthews correlation coefficient (MCC) [11] as evaluation metric to evaluate equally. MCC is calculated as (7) when the number of true positive in the prediction result is $T P$, the number of true negative is $T N$, the number of false positive is $F P$, and the number of false negative is $F N$. The maximum value of MCC is 1 , the minimum value is -1 , and a larger value represents that performance is better.

$$
\mathrm{MCC}=\frac{T P \cdot T N-F P \cdot F N}{\sqrt{(T P+F P)(T P+F N)(T N+F P)(T N+F N)}}
$$

\subsection{Content-based Filtering}

We predicted users' preference for properties by the content-based filtering of 3.3 using top $k_{u}(=1,10,100 \%)$ of similar users and top $k_{i}(=1,10,100 \%)$ of similar properties in the train set. Fig. 4 shows the result of this prediction.

The best performance was obtained when both $k_{u}$ and $k_{i}$ was $10 \%$. The result shows that if $k_{u}$ is too small, it is difficult to predict accurately because the number of users in the train set that can be referred to is reduced. Conversely, if $k_{u}$ is too large, it is equally difficult to predict accurately because we refer to users in the train set whose attributes are not quite similar with each user in the test set. Moreover, regarding $k_{i}$, when $k_{u}$ is small $\left(k_{u}=1,10 \%\right)$, it is better to choose a value as $k_{i}$ which is not too small and not too large in the same way, but when $k_{u}$ is $100 \%$, which means using the information of all users in the train set, it is likely better to use the information of all properties in the train set as well.

\subsection{MLP}

We predicted users' preference for properties by the MLP of 3.4 and compared the following three types of input:

w/o image feature:

Using only attribute data of users and properties
Table 1: Prediction results of users' preference for properties by the MLP

\begin{tabular}{|l|l|l|l|}
\hline Input & $\begin{array}{l}\text { w/o image fea- } \\
\text { ture }\end{array}$ & $\begin{array}{l}\text { w/ image feature } \\
\text { (pre-trained) }\end{array}$ & $\begin{array}{l}\text { w/ image feature } \\
\text { (fine-tuned) }\end{array}$ \\
\hline MCC & 0.127 & 0.142 & $\mathbf{0 . 1 4 9}$ \\
\hline Accuracy & 0.612 & 0.616 & $\mathbf{0 . 6 1 9}$ \\
\hline Precision & 0.439 & 0.446 & $\mathbf{0 . 4 5 1}$ \\
\hline Recall & 0.396 & 0.418 & $\mathbf{0 . 4 2 3}$ \\
\hline $\begin{array}{l}\text { Confusion } \\
\text { matrix }\end{array}$ & & \\
$\left(\begin{array}{ll}T N & F P \\
F N & T P\end{array}\right)$ & $\left(\begin{array}{cc}20843 & 7802 \\
9281 & 6094\end{array}\right)$ & $\left(\begin{array}{cc}20678 & 7967 \\
8954 & 6421\end{array}\right)$ & $\left(\begin{array}{cc}20725 & 7920 \\
8866 & 6509\end{array}\right)$ \\
\hline
\end{tabular}

Table 2: Prediction results of users' preference for properties by the hybrid filtering

\begin{tabular}{|l|l|l|l|}
\hline Input & $\begin{array}{l}\text { w/o image fea- } \\
\text { ture }\end{array}$ & $\begin{array}{l}\text { w/ image feature } \\
\text { (pre-trained) }\end{array}$ & $\begin{array}{l}\text { w/ image feature } \\
\text { (fine-tuned) }\end{array}$ \\
\hline MCC & 0.150 & 0.159 & $\mathbf{0 . 1 6 6}$ \\
\hline Accuracy & 0.600 & 0.604 & $\mathbf{0 . 6 0 7}$ \\
\hline Precision & 0.437 & 0.442 & $\mathbf{0 . 4 4 6}$ \\
\hline Recall & 0.500 & 0.507 & $\mathbf{0 . 5 1 4}$ \\
\hline Confusion & & & \\
matrix & & & \\
$\left(\begin{array}{ll}T N & F P \\
F N & T P\end{array}\right)$ & $\left(\begin{array}{cc}18748 & 9897 \\
7693 & 7682\end{array}\right)$ & $\left(\begin{array}{cc}18796 & 9849 \\
7579 & 7796\end{array}\right)$ & $\left(\begin{array}{cc}18823 & 9822 \\
7479 & 7876\end{array}\right)$ \\
\hline
\end{tabular}

w/ image feature (pre-trained):

Using attribute data of users and properties, and deep features extracted from floor plan images by the pre-trained model of ImageNet [9]

w/ image feature (fine-tuned):

Using attribute data of users and properties, and deep features extracted from floor plan images by the fine-tuned model in 3.4

Table 1 shows the result of the prediction.

In the three types of input used in this study, the performance of the MLP was the best when we used deep features extracted from the floor plan images by the fine-tuned model in 3.4 as a part of the input. The performance of the MLP is improved by adding deep features of floor plans to input. Therefore, considering floor plans seems to be effective in predicting the users' preference for properties. In addition, the performance of the MLP is higher with the fine-tuned model than that with the pre-trained model of ImageNet [9] as the extractor of deep features of floor plans. Hence, it is considered that better deep features can be extracted by finetuning so that feature of the floor plans can be expressed well.

\subsection{Hybrid Filtering}

We predicted users' preference for properties by the hybrid filtering of 3.5 and compared the same three types of input as 4.3. Table 2 shows the result of the prediction.

Among all the prediction systems mentioned above, the best performance was achieved by hybrid filtering that added deep features extracted from floor plan images by the fine-tuned model into 
the input. As a comparison among the three types of input with hybrid filtering, the characteristics of the prediction performance is similar to 4.3. When hybrid filtering is compared with the MLP, we can see that all the prediction performances are considerably improved. Therefore, it is considered that by using content-based filtering and MLP in combination, there is an effect of improving generalization performance, like that of an ensemble in machine learning.

\section{CONCLUSION}

In this study, for developing a recommender system for special items that are not mass-produced, we predicted users' preference for real estate properties using three methods: content-based filtering, which uses similarities of both users and items; MLP, which adds deep features extracted from floor plan images using $\mathrm{CNN}$ into input; and hybrid filtering, which is a combination of the first two methods. The best performance was achieved with hybrid filtering which added deep features extracted from floor plan images by the fine-tuned model into the input.

There are two limitations of this research work. First, in this research, we used the method proposed by Takada et al. [16] to extract deep features of floor plans; however, we have not compared it with other methods. For example, Liu et al. [10] succeeded in converting a rasterized floor plan image into a vector graphics representation. This method is composed of multiple stages, and we can apply their discriminative network that they use for extracting junction layer from input images, for our proposed method. Second, when using our proposed method for property recommendation, the speed of processing is important. However, content-based filtering used in this study is likely to be computationally intensive; thus, we must make it more efficient for actual property recommendation. It is also time consuming to read floor plan images and pass them through the extractor to obtain deep features every time when recommending properties; thus, we must resolve it, e.g., by extracting deep features in advance. If we can solve these problems, we can use our proposed method for actual property recommendation; thus, we designate these as future tasks.

\section{ACKNOWLEDGMENTS}

This work was partly supported by the Grants-in-Aid for Scientific Research (no. 26700008) from JSPS and JST-CREST (JPMJCR1686).

\section{REFERENCES}

[1] John S Breese, David Heckerman, and Carl Kadie. 1998. Empirical analysis of predictive algorithms for collaborative filtering. In Proceedings of the Fourteenth Conference on Uncertainty in Artificial Intelligence (UAI). Morgan Kaufmann Publishers Inc., 43-52.

[2] Simon Func. [n. d.]. Netflix Update: Try This at Home. ([n. d.]). http://sifter.org/simon/journal/20061211.html.

[3] David Goldberg, David Nichols, Brian M. Oki, and Douglas Terry. 1992. Using Collaborative Filtering to Weave an Information Tapestry. Commun. ACM 35, 12 (Dec. 1992), 61-70. https://doi.org/10.1145/138859.138867

[4] Toshihiro Hanazato, Yusuke Hirano, and Makoto Sasaki. 2005. Syantic analysis of large size condominium units supplied in the tokyo metroporitan area. Fournal of Structural and Construction Engineering 70, 591 (2005), 9-16. https://doi.org/10.3130/aija.70.9_5

[5] Kaiming He, Xiangyu Zhang, Shaoqing Ren, and Jian Sun. 2015. Deep Residual Learning for Image Recognition. CoRR abs/1512.03385 (2015). arXiv:1512.03385 http://arxiv.org/abs/1512.03385

[6] Balázs Hidasi, Alexandros Karatzoglou, Linas Baltrunas, and Domonkos Tikk. 2015. Session-based Recommendations with Recurrent Neural Networks. CoRR abs/1511.06939 (2015). arXiv:1511.06939 http://arxiv.org/abs/1511.06939

[7] Sergey Ioffe and Christian Szegedy. 2015. Batch Normalization: Accelerating Deep Network Training by Reducing Internal Covariate Shift. CoRR abs/1502.03167 (2015). arXiv:1502.03167 http://arxiv.org/abs/1502.03167

[8] Yehuda Koren, Robert Bell, Chris Volinsky, et al. 2009. Matrix factorization techniques for recommender systems. Computer 42, 8 (2009), 30-37.

[9] Alex Krizhevsky, Ilya Sutskever, and Geoffrey E Hinton. 2012. ImageNet Classification with Deep Convolutional Neural Networks. In Advances in Neural Information Processing Systems 25, F. Pereira, C. J. C. Burges, L. Bottou, and K. Q. Weinberger (Eds.). Curran Associates, Inc., 1097-1105.

[10] Chen Liu, Jiajun Wu, Pushmeet Kohli, and Yasutaka Furukawa. 2017. Raster-ToVector: Revisiting Floorplan Transformation. In The IEEE International Conference on Computer Vision (ICCV).

[11] B.W. Matthews. 1975. Comparison of the predicted and observed secondary structure of T4 phage lysozyme. Biochimica et Biophysica Acta (BBA) - Protein Structure 405, 2 (1975), 442 - 451. https://doi.org/10.1016/0005-2795(75)90109-9

[12] Kohei Ohara, Toshihiko Yamasaki, and Kiyoharu Aizawa. 2016. An Intuitive System for Searching Apartments using Floor Plans and Areas of Rooms. The 78th national convention of IPS7 (2016). (in Japanese).

[13] D. M. PENNOCK. 2000. Collaborative filtering by personality diagnosis : A hybrid memory and model-based approach. Proceedings of the Sixteenth Conference on Uncertainty in Artificial Intelligence (UAI) (2000). http://ci.nii.ac.jp/naid/10024678257/

[14] Karen Simonyan and Andrew Zisserman. 2014. Very Deep Convolutional Networks for Large-Scale Image Recognition. CoRR abs/1409.1556 (2014). arXiv:1409.1556 http://arxiv.org/abs/1409.1556

[15] Nitish Srivastava, Geoffrey E Hinton, Alex Krizhevsky, Ilya Sutskever, and Ruslan Salakhutdinov. 2014. Dropout: a simple way to prevent neural networks from overfitting. Fournal of machine learning research 15, 1 (2014), 1929-1958.

[16] Yuki Takada, Naoto Inoue, Toshihiko Yamasaki, and Kiyoharu Aizawa. 2018. Similar Floor Plan Retrieval Featuring Multi-Task Learning of Layout Type Classification and Room Presence Prediction. IEEE International Conference on Consumer Electronics (ICCE) (2018), 931-936.

[17] Atsushi Takizawa, Kazuma Yoshida, and Naoki Kato. 2008. Applying graph mining to rent analysis considering room layouts. Fournal of Environmental Engineering 73, 623 (2008), 139-146. https://doi.org/10.3130/aije.73.139

[18] G.-R. XUE. 2005. Scalable collaborative filtering using cluster-based smoothing. Proc. 28th Annual ACM SIGIR Conf. on Research and Development in Information Retrieval (2005). http://ci.nii.ac.jp/naid/10020213129/ 